\title{
Words matter: after more than a century 'schizophrenia' needs rebranding
}

\author{
Antonio Lasalvia
}

\begin{abstract}
SUMMARY
Professionals, patients and patient organisations have advocated for the renaming of 'schizophrenia', seeing the term as misleading, stigmatising and a barrier to specialised care. In countries where a name change has occurred (e.g. Japan), the advantages of renaming seem to far outweigh disadvantages. Nevertheless, a name change would be a long and complex process that would not be useful if it were not accompanied by parallel changes in legislation, services and the education of professionals and the public.
\end{abstract}

\section{DECLARATION OF INTEREST}

None.

\begin{abstract}
'When I use a word' Humpty Dumpty said, in rather a scornful tone, 'it means just what I choose it to mean neither more nor less.' 'The question is,' said Alice, 'whether you can make words mean so many different things.' 'The question is,' said Humpty Dumpty, 'which is to be master - that's all.'
\end{abstract}

(Lewis Carroll, Through the Looking Glass, 1871)

\section{Schizophrenia as metaphor}

In recent years, both patient organisations and mental health professionals around the world have started asking for a change of the term 'schizophrenia', because of its stigmatising connotations. In all cultures throughout the world, schizophrenia is the psychiatric condition most strongly associated with stereotypes, prejudice and discrimination. It has a far worse public acceptance than any other mental disorder (Reavley 2011), as it is dominated by the stereotypes of unpredictability and dangerousness. Fear, stigmatisation and social rejection of people with schizophrenia are strongly rooted in the population and have steadily increased over the past 20 years (Angermeyer 2013). This negative view is sustained by a mass media that inappropriately uses the term 'schizophrenic' to describe any incoherent, contradictory or deviant behaviour, or criminal, violent or dangerous individuals (Clement 2008). Thus, 'schizophrenia' nowadays seems to be commonly used as a metaphor, especially in political articles and in culture, entertainment and sport sections (Magliano 2011).

Metaphorical references to an illness can conjure up negative, disheartening associations and, when commonly accepted, contribute to the social rejection and degradation of well-being of individuals with that illness. In Illness as Metaphor, Susan Sontag (1989) posited that feared illnesses are used as metaphors - for example, tuberculosis in the 19th century and cancer in the 20th. Sontag's work suggests that misuse of metaphors is a reflection of ongoing stigmatising beliefs; thus, metaphors involving mental disorders are likely to appear more often than those involving less stigmatised conditions (Duckworth 2003). Over the past century, psychiatry has made Humpty Dumpty's fundamental error, believing it possible to remain the master of its own words and to control their usage by, and connotations for, others. However, words tend to take on a life of their own, often becoming ambiguous and misleading in the process. And once an incorrect usage acquires the tenure of long and wide acceptance, it can be very difficult to control or to replace (Frances 2015). A similar process seems to have specifically occurred for schizophrenia.

\section{Schizophrenia, a contested word}

It is no wonder that the term schizophrenia has been increasingly contested by patients and families. In October 2006, on the eve of World Mental Health Day, the UK patient organisation Hearing Voices Network and supporters of Asylum magazine launched the Campaign for the Abolition of the Schizophrenia Label (CASL) (Hammersley 2006). This campaign was based on the assumption that schizophrenia is an 'unscientific' concept that has outlived any usefulness it might once have claimed and that it is extremely damaging to those to whom it is applied. CASL had a great impact on the British media (e.g. BBC News 2006; Boseley 2006; Daily Mail 2006). In April 2009, CASL launched a petition addressed to the UK prime minister calling for the abolition of the term schizophrenia. In North America, service user movements have
CLINICAL REFLECTION
Antonio Lasalvia is a consultant psychiatrist in the Verona University Integrated Hospital (Azienda Ospedaliera Universitaria Integrata (AOUI) di Verona) and head of the 4th Community Mental Health Service of Verona ULSS9 Scaligera.

Correspondence Dr Antonio Lasalvia, U.O.C. Psichiatria, Azienda Ospedaliera Universitaria Integrata (AOUI) di Verona, Policlinico 'G.B. Rossi', P.le Scuro, 1037134 - Verona, Italy. Email: antonio.lasalvia@univr.it

Copyright and usage (C) The Royal College of Psychiatrists 2018 
been raising voices against the label 'schizophrenia'. In Canada, Bill MacPhee, who had been diagnosed with schizophrenia over 25 years earlier and was publisher of SZ Magazine, launched a campaign to change the name of schizophrenia to MacPhee syndrome. His campaign, using crowdfunding to sustain a speaking tour across North America, petitioned the American Psychiatric Association task force to change the name 'schizophrenia' in the forthcoming DSM-5.

Even from within the psychiatric field there have been calls to change the term. According to some clinicians and researchers the word 'schizophrenia' is misleading, since 'split brain' has nothing to do with this disorder (Kingdon 2013). The term 'schizophrenia' in fact provides no information about the fundamental nature (e.g. pathophysiology) and psychopathological structure of the disorder (Maj 1998). The word is also of limited clinical utility, since it gives no specific information on either prevention (Boyle 2004) or treatment (van Os 2009).

A public endorsement for changing the name of schizophrenia was made in 2006 by Sir Robin Murray, Professor of Psychiatry at the Institute of Psychiatry in London (Murray 2006). In 2011, on the 100th anniversary of the introduction of 'schizophrenia' in the scientific literature, a group of British psychiatrists, mostly working for the National Health Service, proposed that the best way to celebrate the event was 'to consign schizophrenia to history' (Critical Psychiatry Network 2011). More recently, Jim van Os, Professor of Psychiatry at Maastricht University, concluded in a provocative editorial in the $B M J$ that 'ICD-11 should remove the term "schizophrenia" , (van Os 2016); his position was echoed by Robin Murray (2017), who maintained that 'I expect to see the end of the concept of schizophrenia soon [...]. Presumably this process will accelerate, and the term schizophrenia will be confined to history, like "dropsy" '.

\section{Renaming schizophrenia}

Some instances of renaming schizophrenia have already taken place. Japan was the first country to change the name, following a long process that began in 1993 with an initiative by the national family organisation Zenkaren. In 2002, the Japanese Society of Psychiatry and Neurology (JSPN) proposed that the old term 'schizophrenia' be replaced with 'integration dysregulation syndrome'. This new name was officially recognised by the Japanese Ministry of Health and Welfare in 2005. The new term refers to the stress-vulnerability model of the disorder, underlining that it is treatable and recovery is possible with a combination of advanced pharmacotherapy and psychosocial intervention. In South Korea in 2011, the Korean Neuropsychiatric Association and Korean Society for Schizophrenia Research renamed 'schizophrenia' as 'attunement disorder', and in 2012 a law including the new term was passed by the National Assembly. In Hong Kong and Taiwan there have been official proposals to change the term 'schizophrenia' (Sartorius 2014).

\section{The evidence so far: advantages}

Research evidence from the countries where the name change has occurred suggests that the advantages of renaming schizophrenia far outweigh the disadvantages (Lasalvia 2015). The Japanese experience shows that an early effect of the renaming was improvement in clinician-patient-family communication, with a significant increase in the number of patients and carers informed about the diagnosis, prognosis and available interventions (Sato 2006). Another positive effect of renaming was the reduction of stigma related to the condition over the medium (Takahashi 2009; Sugihara 2013) and the long term (Koike 2015), and across different age groups (Koike 2017).

Thus, a new term for schizophrenia that avoids stigmatising connotations seems to be more acceptable for both patients and professionals and would facilitate patient-clinician communication. A name change would also improve the public image of the disorder and the people who suffer from it, thus reducing stigma. This would encourage helpseeking and service uptake by patients and, in turn, it would help them to take full advantage of treatment provided. A name change might also be welcomed by researchers, since it could stimulate the scientific community to reformulate and reconceptualise the condition. A new name might also improve funding for both research and services. However, a name change is a complex process whose impact should be considered in the light of the public representation of the condition by the mass media. The evidence in this regard is conflicting. Koike (2016), in a study examining four major Japanese newspapers and one TV news programme over the period 1985-2013, found little effect of the name change on newspaper articles; on the other hand, another study investigating newspaper coverage of schizophrenia over 20 years (in the decades before and after renaming) found a reduction in stigmatising depiction of the condition with the new term (Aoki 2016). These findings suggest that article contents in the mass media change slowly, thus indicating that the effect of the name change on stigma in mass media reports should be considered over a longer period. 


\section{... and disadvantages?}

Renaming schizophrenia might also have some problematic consequences. For nearly a century 'schizophrenia' has been an integral part of psychiatric nosology and has been used in research and treatment. The diagnosis of schizophrenia satisfies criteria for a valid diagnostic entity, is conceptualised similarly across the world and shows the highest inter-rater diagnostic reliability among psychiatric diagnoses. Other terms may convey less information about the disorder and this may lead to disagreement among both researchers and professionals. Moreover, a name change may wrongly suggest that some fundamental truth about the condition has been newly discovered (unfortunately, this is not the case); it may be only semantics; it may have the effect of blaming the person rather than the illness; it may have only a temporary effect (stigma will soon attach to the new term).

\section{The process of change}

A central issue is which name should be used to replace schizophrenia. A number of different options have been proposed over the years (Box 1), but none has gained general consensus (Lasalvia 2015). The most conservative option would be to use an eponym (e.g. Bleuler's syndrome), since eponyms are neutral and avoid connotations.

A name change, however, is a long and complex process that requires the involvement of all key stakeholders (i.e. patients, carers, professionals, researchers and the general public) and it should be accompanied by widespread educational and anti-stigma campaigns. What really needs to be changed is the public perception of what is currently known as 'schizophrenia', rather than the name itself. To persuade the general public to be more accepting of people with this illness, it is necessary to make it clear that psychosis arises, in part, from adverse life experiences, that it does not necessarily lead to violence and that recovery is possible. However, renaming schizophrenia may be an initial step forward.

\section{Changed name, changed perceptions?}

There have been some important precedents for such a change in both medicine and psychiatry: for example from leprosy ('leper') to Hansen's disease (1952); from Mongolism ('mongoloid') to Down syndrome (1965); from manic-depressive illness ('manic') to bipolar disorder (1980); and, more recently, from mental retardation ('retarded') to intellectual disability (2013). Terms previously in common use, such as 'mongoloid', 'manic' and 'retarded', have all been changed with success. This process has probably not completely eradicated the stigma attached to these conditions, but has provided more generally acceptable terms. Certainly any return to the use of the previous terminology is not contemplated and its use is now seen as insulting and derogatory. Why could the same not happen for 'schizophrenia'?

\section{Conclusions}

The language we use greatly influences the way we think. The Greek word $\lambda$ óros (logos) took on a dual meaning - as 'word', 'speech', 'discourse' and as 'reasoning', 'thought' - thus indicating the strong relationship (a sort of overlap) between the process of naming and the act of thinking. The language we use is important not so much in its own right but because it reflects - and also shapes - the way we think about things. Recent studies in linguistics and cognitive science have shown that word choice and language use have direct influences on the speaker's thoughts and actions (Boroditsky 2011). Word choice and the context to which the words are attributed can foster stigma and prejudice towards people with mental illness, trivialising serious conditions and their accompanying experiences. Anti-stigma campaigns, designed to raise public awareness of the stigmatising of mental illness, have in recent years focused on bringing public attention to the negative impact of their choice of words (Hwang 2016). It is not just a matter of semantics or being 'politically correct': the language we use reflects what we think and how we feel about a given condition and the people

\section{BOX 1 'Schizophrenia': some alternatives}

In Japan and South Korea 'schizophrenia' is officially renamed 'integration dysregulation syndrome' and 'attunement disorder' respectively. Suggested alternative names from other countries have included the following.

In 1990, lan Falloon, Professor of Psychiatry at the University of Auckland and a board member of the World Association for Psychosocial Rehabilitation (WAPR), suggested replacing 'schizophrenia' with 'Bleuler's and Kretschmer's syndrome' or 'Schneider's syndrome', with the advantage of removing prognostic implications. Michael Madianos, former president of the WAPR, suggested 'dysphrenia', from the Greek dys and phrene (dysfunction of the mind) (Madianos 2008).

In 2006, Sir Robin Murray, Professor of Psychiatry at the Institute of Psychiatry in London, proposed replacing schizophrenia with 'dopamine dysregulation disorder' (Murray 2006).

In 2009, Jim van Os, Professor of Psychiatry at Maastricht University, proposed replacing schizophrenia with 'salience syndrome' (van Os 2009). 
suffering from it; and this in turn has real consequences in people's lives:

'The metaphoric trappings that deform the experience of having a disease [...] have very real consequences: they inhibit people from seeking treatment early enough, or from making a greater effort to get competent treatment. The metaphor and myths, I am convinced, kill' (Sontag 1989).

\section{References}

Angermeyer MC, Matschinger H, Schomerus G (2013) Attitudes towards psychiatric treatment and people with mental illness: changes over two decades. British Journal of Psychiatry, 203: 146-51.

Aoki A, Aoki Y, Goulden R, et al (2016) Change in newspaper coverage of schizophrenia in Japan over 20-year period. Schizophrenia Research, 175: 193-7.

BBC News (2006) Schizophrenia term use 'invalid', BBC News, 9 October (http://news.bbc.co.uk/2/hi/health/6033013.stm). Accessed 30 June 2017.

Boroditsky L (2011) How language shapes thought. Scientific American, 304: 62-5.

Boseley S (2006) Call to wipe out schizophrenia as catch-all tag. Guardian, 10 October (http://www.theguardian.com/news/2006/oct/10/medicineandhealth). Accessed 30 June 2017.

Boyle M (2004) Preventing a non-existent illness? Some issues in the prevention of 'schizophrenia'. Journal of Primary Prevention, 24: 445-68.

Clement S, Foster N (2008) Newspaper reporting on schizophrenia: a content analysis of five national newspapers at two time points. Schizophrenia Research, 98: 178-83.

Critical Psychiatry Network (2011) The Case against Schizophrenia. Critical Psychiatry Network (http://www.criticalpsychiatry.co.uk/index. php?option=com_content\&view=article\&id=46:the-case-against-schizophrenia \&catid=35:documents\&ltemid=56). Accessed 4 July 2017.

Daily Mail (2006) Experts call for ban on schizophrenia 'label'. Daily Mail, 9 October (http://www.dailymail.co.uk/health/article-409472/Expertsban-schizophrenia-label.html). Accessed 30 June 2017.

Duckworth K, Halpern JH, Schutt RK, et al (2003) Use of schizophrenia as a metaphor in US newspapers. Psychiatric Services, 54: 1402-4.

Frances A (2015) Can we replace misleading terms like 'mental illness,' 'patient,' and 'schizophrenia'. Huffington Post: The Blog, 4 June (http:// www.huffingtonpost.com/allen-frances/can-we-replace-misleadingterms-like-mental-illness-patient-schizophrenia_b_7000762.html). Accessed 30 June 2017.

Hammersley P, McLaughlin T (2006) Campaign for the Abolition of Schizophrenia Label. Asylum Magazine (http://www.asylumonline.net/ resources/campaign-for-the-abolition-of-schizophrenia-label). Accessed 30 June 2017

Hwang JD, Hollingshead K (2016) Crazy, mad, nutters: the language of mental health. In Proceedings of the 3rd Workshop on Computational Linguistics and Clinical Psychology: From Linguistic Signal to Clinical
Reality (eds K Hollingshead, L Ungar): 52-62. Association for Computational Linguistics.

Kingdon D, Taylor L, Kinoshita $Y$ (2013) Changing name: changing prospects for psychosis. Epidemiology and Psychiatric Sciences, 22: 297-301.

Koike S, Yamaguchi S, Ojio Y, et al (2015) Long-term effect of a name change for schizophrenia on reducing stigma. Social Psychiatry and Psychiatric Epidemiology, 50: 1519-26.

Koike S, Yamaguchi S, Ojio Y, et al (2016) Effect of name change of schizophrenia on mass media between 1985 and 2013 in Japan: a text data mining analysis. Schizophrenia Bulletin, 42: 552-9.

Koike S, Yamaguchi S, Ohta K, et al (2017) Mental-health-related stigma among Japanese children and their parents and impact of renaming of schizophrenia. Psychiatry and Clinical Neurosciences, 71: $170-9$.

Lasalvia A, Penta E, Sartorius N, et al (2015) Should the label 'schizophrenia' be abandoned? Schizophrenia Research, 162: 276-84.

Madianos MG (2008) The myth of the splitting of the brain or the need for renaming schizophrenia. World Association of Psychiatric Rehabilitation Bulletin, 25: 3-5.

Magliano L, Read J, Marassi R (2011) Metaphoric and non-metaphoric use of the term 'schizophrenia' in Italian newspapers. Social Psychiatry Psychiatric Epidemiology, 46: 1019-25.

Maj M (1998) Critique of the DSM-IV operational diagnostic criteria for schizophrenia. British Journal of Psychiatry, 172: 458-60.

Murray RM (2006) Schizophrenia term use 'invalid'. BBC News, 9 October. (http://news.bbc.co.uk/2/hi/health/6033013.stm). Accessed 30 June 2017.

Murray RM (2017) Mistakes I have made in my research career. Schizophrenia Bulletin, 43: 253-6.

Reavley NJ, Jorm AF (2011) Stigmatising attitudes towards people with mental disorders: findings from an Australian National Survey of Mental Health Literacy and Stigma. Australian and New Zealand Journal of Psychiatry, 45: 1086-93.

Sartorius N, Chiu H, Heok KE, et al (2014) Name change for schizophrenia. Schizophrenia Bulletin, 40: 255-8.

Sato M (2006) Renaming schizophrenia: a Japanese perspective. World Psychiatry, 5: 53-5.

Sontag S (1989) AIDS and its Metaphors. Farrar, Straus \& Giroux.

Sugihara G, Takei N (2013) Renaming schizophrenia coupled with proper public education is an optimal way to overcome stigma. Psychological Medicine, 43: 1557-8.

Takahashi H, Ideno T, Okubo S, et al (2009) Impact of changing the Japanese term for 'schizophrenia' for reasons of stereotypical beliefs of schizophrenia in Japanese youth. Schizophrenia Research, 112: $149-52$

van Os J (2009) 'Salience syndrome' replaces 'schizophrenia' in DSM-5 and ICD-11: psychiatry's evidence-based entry into the 21st century? Acta Psychiatrica Scandinavica, 120: 363-72.

van Os J (2016) 'Schizophrenia' does not exist. BMJ, 352: i375. 